\title{
Magnetic control over the topology of supramolecular rod networks
}

\author{
Vincent Marichez ${ }^{[a]}$, Akihiro Sato ${ }^{[a]}$, Peter Dunne ${ }^{[a]}$, Jorge Leira-Iglesias ${ }^{[a]}$, Georges J.M. Formon ${ }^{[a]}$, \\ Michaela K. Schicho ${ }^{[a]}$, Isja de Feijter ${ }^{[b]}$, Pascal Hébraud ${ }^{[c]}$, Matthieu Bailleul[ ${ }^{[c]}$, Pol Besenius ${ }^{[d]}$, M. \\ Venkatesan $^{[e]}$, J.M.D. Coey ${ }^{[e]}$, E.W. Meijer ${ }^{[b]}$, and Thomas M. Hermans ${ }^{\star[a]}$
}

\begin{abstract}
Understanding and controlling supramolecular polymerization are of fundamental importance to create advanced materials and devices. Many stimuli have been explored in the past decades, but magnetic fields and field gradients have received little attention. This is because magnets do not provide enough magnetic energy to overcome thermal noise at the single molecule level. Here we show that significant changes in network topology of $\mathrm{Gd}^{3+}$. decorated supramolecular polymer rods can nevertheless be observed using magnetic fields of order $1 \mathrm{~T}$ at room temperature. The structure of the rod networks is influenced during a slow diffusive process over a timescale of hours by the anisotropy of the demagnetizing field. Our approach opens opportunities to control and tune structure formation of many supramolecular and coordination polymers using a variety of rare earth or other paramagnetic ions.
\end{abstract}

Supramolecular polymers consist of monomers held together by reversible non-covalent interactions, and have been extensively studied in the past decades. ${ }^{[1]}$ Their properties are commonly controlled by external stimuli: ${ }^{[2]}$, such as temperature ${ }^{[1]}$ enzymes ${ }^{[3-5]}$, mechanical forces ${ }^{[6,7]}$, light ${ }^{[8-10]}, \mathrm{pH}^{[11-13]}$ and redox potentia ${ }^{[14,15]}$. In addition, strong electric ${ }^{[16]}$, magnetic ${ }^{[17]}$ or flowfields ${ }^{[18,19]}$ have been used to control the growth and orientation of supramolecular polymers. In contrast to colloidal systems where weak magnetic fields can guide assembly of ferrimagnetic nanocrystals, ${ }^{[20]}$ large $10-30 \mathrm{~T}$ fields are typically needed to observe any effects on paramagnets at the molecular scale.

In recent years, however, several studies have reported that magnetic fields $<2 \mathrm{~T}$ can significantly change the assembly of molecules decorated with chelated paramagnetic ions. Specifically, Polarz et al., used a $\mathrm{C}_{10}$-DOTA surfactant chelating $\mathrm{Dy}^{3+}$, which formed $\mathrm{mm}$-size self-assembled dumbbells that could be aligned using the stray field of $\mathrm{Nd}_{2} \mathrm{Fe}_{14} \mathrm{~B}$ magnets $(<1 \mathrm{~T}) .{ }^{[21]}$ Yue et al., were able to orient tetrabromoferrate-modified cylindrical block-copolymer phases using just $\sim 0.35$ T. ${ }^{[22]}$ Furthermore, Schefer et al., showed that polysaccharides

[a] Dr. V. Marichez, Dr. A. Sato, Dr. P. Dunne, Dr. J. Leira-Iglesias, G.J.M. Formon, M.K. Schicho, Prof. Dr. T.M. Hermans Université de Strasbourg, CNRS, UMR7140, Strasbourg (France) E-mail: hermans@unistra.fr

[b] Dr. I. de Feijter, Prof. Dr. E.W. Meijer Laboratory of Macromolecular and Organic Chemistry Eindhoven University of Technology P.O. Box 513, 5600 MB Eindhoven (The Netherlands)

[c] Dr. P. Hébraud, Dr. M. Bailleul Institut de Physique et Chimie des Materiaux de Strasbourg, UMR 7504 CNRS-Université de Strasbourg 23 rue du Loess, 67034 Strasbourg (France)

[d] Prof. Dr. P. Besenius Department of Chemistry Johannes Gutenberg-University Mainz Duesbergweg 10-14, 55128 Mainz (Germany)

[e] Dr. M. Venkatesan, Prof. Dr. J.M.D. Coey Physics Department Trinity College, Dublin 2 (Ireland) chelated with ferric ions can stiffen 1.5-fold in a $1.1 \mathrm{~T}$ magnetic field. These results appear surprising considering the magnetic energies involved. In a uniform $1 \mathrm{~T}$ field; the magnetic energy, $U_{\mathrm{m}}$ $=-1 / 2 m B$, of a single paramagnetic $\mathrm{Gd}^{3+}$ ion at room temperature, is $-3 \cdot 10^{-25} \mathrm{~J}^{[23]}$, four orders of magnitude smaller than the thermal energy at room temperature $E_{T}=\mathrm{k}_{\mathrm{B}} T=4 \cdot 10^{-21} \mathrm{~J}$.

Here, we show that magnetic fields of $1-2 \mathrm{~T}$ can change the topology of supramolecular networks, which we quantify by measuring their fractal dimension. The effect is attributed to an anisotropic magnetic term in the Gibbs free energy. Specifically, we use a well-studied $C_{3}$-symmetrical benzene-1,3,5tricarboxamide (BTA) derivative containing three 1,4,7,10tetraazacyclododecane-N,N',N",N"'-tetraacetic acid (DOTA) groups (Fig. 1b), which as previously shown self-assembles into rods of $\sim 6 \mathrm{~nm}$ in diameter and lengths of hundreds of $\mathrm{nm}$ depending on the total concentration (see $K_{1}$ in Fig. 1a). ${ }^{[24,25]}$ The rods assemble further into supramolecular networks (see $K_{2}$ in Fig. $1 \mathrm{a})$, which as we will show in the current work, respond to magnetic fields if paramagnetic $\mathrm{Gd}^{3+}$ is chelated. For diamagnetic Y(III)-DOTA-BTA no changes are observed (see below).

Light scattering provides a detailed insight into the different structures and networks in aqueous solution. Figure 1c shows the measured polarized electric field autocorrelation functions $g_{V V}^{(1)}(q, t)$ at a scattering wavevector $q=0.0288 \mathrm{~nm}^{-1}$, which are well represented by a distribution of relaxation times $G(\tau)$ obtained from the inverse Laplace transformation. ${ }^{[26]}$

Two distinct relaxation processes $\mathbf{P 1}$ and $\mathbf{P} 2$ were found (shown as solid lines underneath the correlation functions in the figure). P1 is a fast process and P2 is a slow one, both having $q^{2}$-dependence. That is, both processes are diffusive and their hydrodynamic radii $R_{H}$ can be calculated from the diffusion constant $D_{\mathrm{H}}=\Gamma \cdot q^{-2}$ (where $\Gamma$ is the decay constant), using the Stokes-Einstein equation $R_{H}=k_{B} T / 6 \pi \eta D_{H}$ (where $\eta$ is the dynamic viscosity).

In figure $1 \mathrm{~d}$ we can see that Gd(III)-DOTA-BTA forms rods with a diameter of $6 \mathrm{~nm}$ in agreement with our prior studies ${ }^{[24,25]}$. We can therefore convert the values of $R_{H}$ of the individual rods (P1) by fixing the diameter and fitting rod lengths using the Tirado model ${ }^{[27]}$. This analysis leads to a rod length of $\sim 245 \mathrm{~nm}$ for Gd(III)-DOTA-BTA and 120 nm for Y(III)-DOTA-BTA (see Table 1).

Since we are dealing with rods in $\mathbf{P} 1$ and networks of rods in P2, we tried to obtain VH (Vertical-Horizontal) polarized electric field autocorrelation functions $g_{V H}^{(1)}(q, t)$, which unfortunately were unresolved for both, even though for P1 of Gd(III)-DOTA-BTA the $q L>5^{[28]}$. Nonetheless, static light scattering intensities of both Y(III)-DOTA-BTA P2 and Gd(III)-DOTA-BTA P2 follow a $R(q) \propto$ $q^{-d_{F}}$ power law (see Fig. 2a), allowing for the characterization of the network topology using the fractal dimension $d_{F}$ (where the the lower limit of $d_{F}=1$ describes a line, and the upper limit $d_{F}=3$ a sphere). ${ }^{[29]}$ 


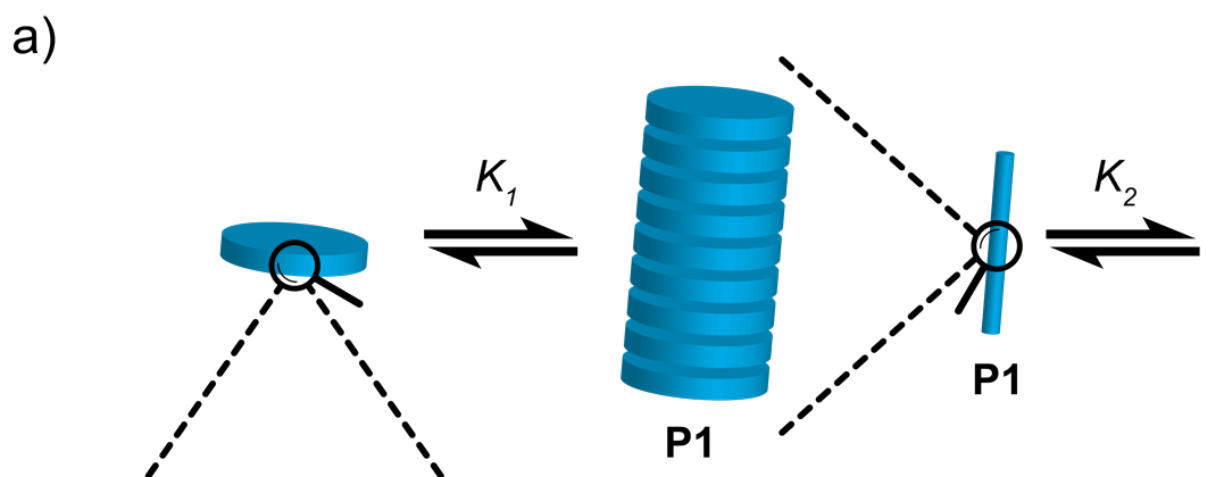

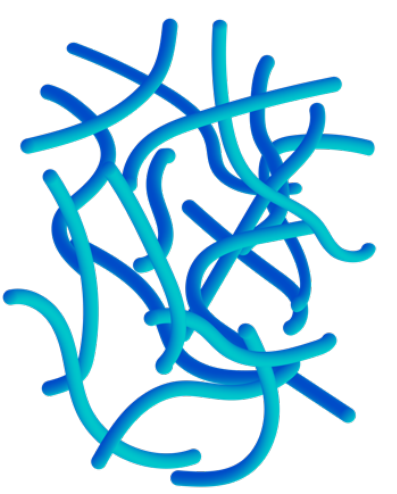

P2

C)
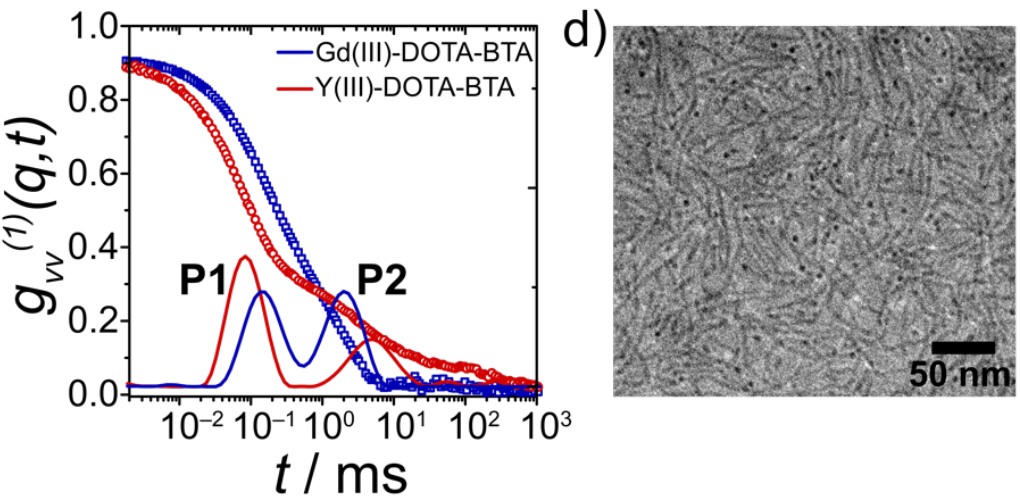

Figure 1. The formation and analysis of networks $\mathbf{P 2}$, consisting of supramolecular rods $\mathbf{P 1}$. a) Scheme depicting equilibria $\left(K_{1}\right.$ and $\left.K_{2}\right)$ that take place during self-assembly of $\mathrm{Gd}(\mathrm{III})$-DOTA-BTA and how the magnetic Gibbs free energies $\left(\Delta G_{m}\right)$ compare to thermal fluctuations at $2 \mathrm{~T}$. For Y(III)-DOTA-BTA the assembly process is similar, but not affected by the applied magnetic field (not shown). b) Molecular structure of Gd(III)-DOTA-BTA and Y(III)-DOTA-BTA. Only 1 of three arms is shown for clarity. c) Polarized field correlation function $g_{v v^{(1)}}(q, t)$ at $100 \mu \mathrm{M}$ concentration in $100 \mathrm{mM}^{\mathrm{m}}$ citrate buffer at $\mathrm{pH} 6 \mathrm{measured}$ at $q=0.0288 \mathrm{~nm} \mathrm{~m}^{-1}$, shown by the hollow symbols. The solid lines show the corresponding distribution of relaxation times $G(\tau)$ at this wavevector, showing P1 and P2. d) Cryo-

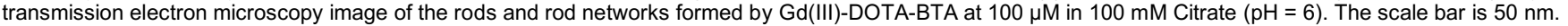

Table 1. Results from light scattering experiments. Hydrodynamic radii $R_{\mathrm{H}}$ of diffusive processes P1 (one-dimensional rods) and P2 (rod networks) extracted from dynamic light scattering data and the respective rod lengths estimated for P1 according to a model by Tirado ${ }^{[27]}$.

\begin{tabular}{llll}
\hline Species & $R_{\mathrm{H}}(\mathbf{P 1}) / \mathrm{nm}$ & $\begin{array}{l}\text { Rod length } \\
\text { (from P1) } / \mathrm{nm}\end{array}$ & $R_{\mathrm{H}}(\mathbf{P 2}) / \mathrm{nm}$ \\
\hline $\begin{array}{l}\text { Gd(III)-DOTA- } \\
\text { BTA }\end{array}$ & $30.5 \pm 4.5$ & 245 (calc.) & $348.2 \pm 69$ \\
$\begin{array}{l}\text { Y(III)-DOTA- } \\
\text { BTA }\end{array}$ & $17.9 \pm 1.5$ & 120 (calc.) & $503.7 \pm 169.6$ \\
\hline
\end{tabular}

In the absence of a magnetic field we find $d_{F}$ of $\mathbf{P} 2$ to be $1.7 \pm 0.2$ which is in agreement with a network of rods present in solution. ${ }^{[30,31]}$ In addition, we performed box-counting analysis of the cryo-TEM images (see Fig. 1d and SI) of Gd(III)-DOTA-BTA leading to $a d_{F}$ of $1.77-1.85$, depending on the choice of threshold for converting to binary information (see $\mathrm{SI}$ ). We are confident that process $\mathbf{P 2}$ is associated with the network topology of rods of Gd(III)-DOTA-BTA.

In the experiments that follow we use $d_{F}$ to quantify the rodlike network topology, and the Rayleigh ratio $R(q)$ to determine the approximate mass contained in the network. The value of $R(q)$ is derived from the static light scattering intensity, by correcting for the refractive index and subtraction of background scattering.

Practically, a light scattering cuvette was placed between the pole pieces of an electromagnet for $1 \mathrm{~h}$, then analyzed by light scattering $(\sim 1 \mathrm{~h})$ without field, and replaced in the magnetic field. This cycle was repeated 6-7 times (12-14 h in total). In Fig. 2b one can see that $R(q)$ for $\mathbf{P 2}$ of $\mathrm{Gd}(\mathrm{III})$-DOTA-BTA increases gradually over time, and reaches a plateau after $10-12 \mathrm{~h}$ that is $20 \%(1 \mathrm{~T})$ or $35 \%(2 \mathrm{~T})$ higher than the original value. The fractal dimension $d_{F}$ changes from $1.7 \pm 0.2$ to $2.2 \pm 0.2(1 \mathrm{~T})$ or $2.6 \pm$ $0.2(2 \mathrm{~T})$ within the first hour and then remains constant for the rest of the experiment (see Fig. S1 in the SI). This implies that the increased magnetic field leads to increasingly dense network structures, ending up at $2 \mathrm{~T}$ with randomly branched clusters ${ }^{[32-}$ 34]. P1 (open symbols, Fig. 2b) is unaffected by the magnetic field as explained below. If the sample is not exposed to the magnetic field, the value of $R(q)$ for $\mathbf{P} 2$ remains constant (see grey dashed box in Fig. 2b). Likewise, if the chelated ion is weakly diamagnetic as in Y(III)-DOTA-BTA, no change is observed. We can therefore conclude that changes in the network topology are only observed when both paramagnetic $\mathrm{Gd}^{3+}$ ions and the magnetic field are present. 


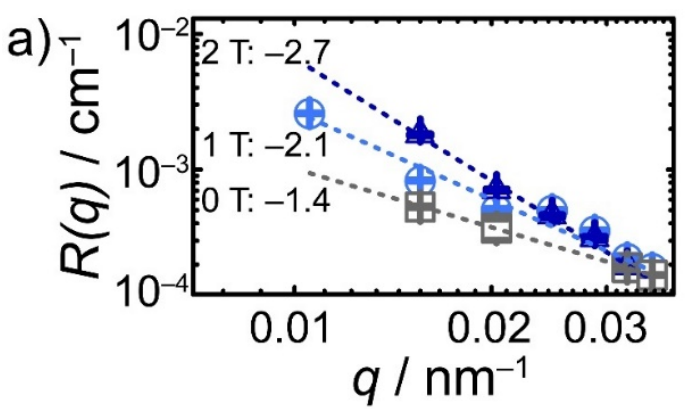

b)

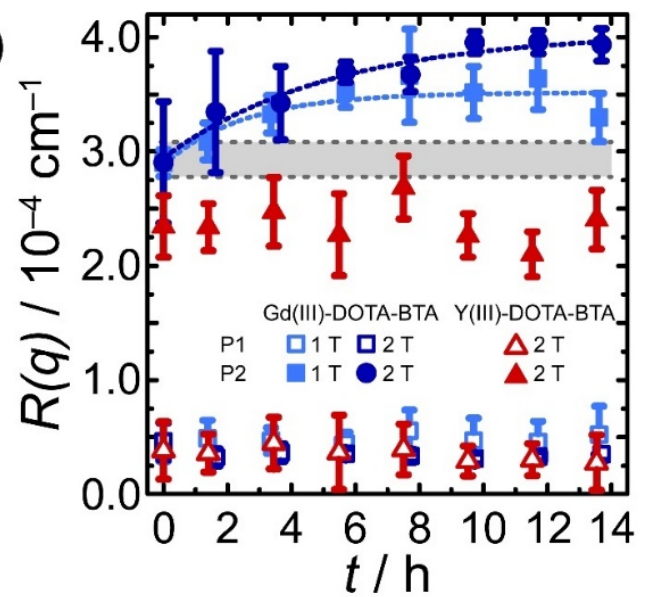

Figure 2. Quantification of rod network topologies upon exposure to magnetic fields. a) Rayleigh ratio $R(q)$, corrected scattered intensity, as a function of wavevector $q$ at normal conditions $(0 \mathrm{~T})$ and with $1 \mathrm{~T}$ and $2 \mathrm{~T}$ applied magnetic field. The slopes of the curves give the fractal dimension of P2 for the different magnetic fields applied. Error bars are standard deviations calculated over 5 measurements. b) Variation of Rayleigh ratio $R(q)$ at $q=0.0288 \mathrm{~nm}^{-1}$ with time for both processes, $\mathbf{P 1}$ and $\mathbf{P 2}$, denoted by hollow and filled symbols respectively. Gd(III)-DOTA-BTA is represented by circles and squares, and Y(III)-DOTA-BTA by triangles. For Y(III)-DOTA-BTA, in red, only the $2 \mathrm{~T}$ data is shown. The gray area delimited by pointed lines represents the control for Gd(III)-DOTA-BTA under no magnetic field (mean and standard deviation). Error bars show standard deviations over 50 measurements.

As mentioned in the introduction, the magnetic energy per ion is generally very much smaller than $k_{B} T$. We therefore checked for interactions between the paramagnetic ions by measuring the susceptibility versus temperature in a SQUID (superconducting quantum interference device) magnetometer. The data (Fig. 3a) show a Curie-Weiss behaviour with a very small negative paramagnetic Curie temperature of $-0.7 \mathrm{~K}$. Antiferromagnetic coupling between the $\mathrm{Gd}^{3+}$ ions in the supramolecular rods is therefore negligible, as is the crystal field interaction for $\mathrm{Gd}^{3+}$, which explained alignment in the $\mathrm{Dy}^{3+}$ compound [21]. We also subjected samples to a constant $5 \mathrm{~T}$ field for up to $12 \mathrm{~h}$ while monitoring the magnetic susceptibility. For Gd(III)-DOTA-BTA, it decreased by $\sim 2 \%$ with respect to the original value $\chi_{0}$ on a timescale commensurate with that found in the light scattering experiments (Fig. $3 b$ ). No changes are observed in the case of Y(III)-DOTA-BTA or a buffer solution (Fig. $3 b)$.
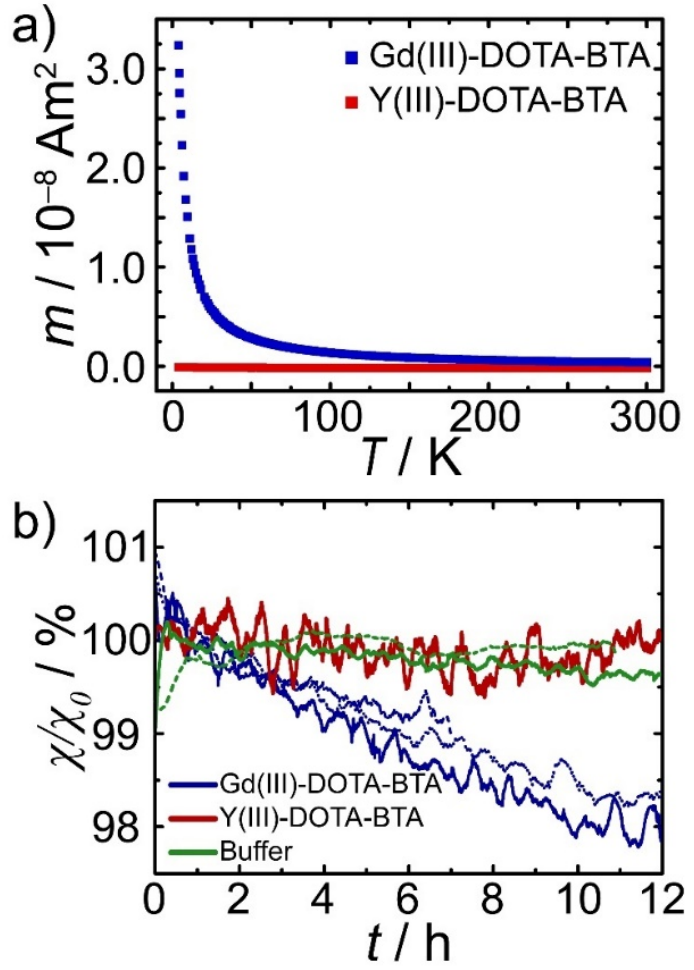

Figure 3. Magnetic characterization of supramolecular rods and networks. a) Temperature-dependent magnetization curve of $\mathrm{Gd}(\mathrm{III})$ and Y(III)-DOTA-BTA in powder form, black and red respectively. $m$ represents the magnetization of the sample in A $\mathrm{m}^{2}$. Gd(III)-DOTA-BTA shows a weak antiferromagnetic coupling $-0.7 \mathrm{~K}$ while the $\mathrm{Y}(\mathrm{III})$ containing molecule showed no paramagnetism. b) Room-temperature time-dependent magnetization of $\mathrm{Gd}(\mathrm{III})$ and $\mathrm{Y}(\mathrm{III})$ DOTA-BTA (blue and red lines respectively) in solution and buffer (in green). The samples were place in the SQUID under $5 \mathrm{~T}$ magnetic field and their magnetization was measured over the course of $12 \mathrm{~h}$. The solid and dotted lines represent different runs.

To evaluate the magnetic influence in comparison to the thermal energy, the magnetic energy of a single paramagnetic monomer can be estimated using a Curie-law dependence by: ${ }^{[23]}$

$$
U_{m, 1}=-\frac{1}{2} m B=\frac{3}{2} \frac{m_{e f f}^{2} B}{3 \mathrm{k}_{\mathrm{B}} T} B=\frac{m_{e f f}^{2} B^{2}}{2 \mathrm{k}_{\mathrm{B}} T}
$$

Where $m$ is the magnetic moment induced by the field $B$ at temperature $T, m_{e f f}$ is the effective atomic paramagnetic moment in $\mathrm{Am}^{2}$ (given in units of Bohr magneton $\mu_{B}$ ). We can define a critical length $L_{c}$ of the supramolecular rod, where the magnetic energy of the object exceeds the thermal energy $\left(\left|U_{m}\right| \geq \mathrm{k}_{\mathrm{B}} T\right)$ :

$$
L_{c}=\frac{d \mathrm{k}_{\mathrm{B}} T}{U_{m, 1}}=2 d\left(\frac{k_{B} T}{m_{e f f} B}\right)^{2}
$$

Here $d$ is the $\pi-\pi$ stacking distance of $0.35 \mathrm{~nm}$ between the monomers in the rod that each contain three $\mathrm{Gd}^{3+}$ ions. ${ }^{[35]}$ The critical length and supramolecular magnetic energy for different paramagnetic ions was calculated (Table 2, and Table S4). In addition, a more detailed model considering the exact positioning of $\mathrm{Gd}^{3+}$ ions within the supramolecular rods, and dipole-dipole interactions, was in quantitative agreement (see $\mathrm{SI}$ ). Overall, this shows that the magnetic energy is smaller than $\mathrm{k}_{\mathrm{B}} T$ for $\mathbf{P 1}$ it can be much larger than $\mathrm{k}_{\mathrm{B}} T$ for $\mathbf{P 2}$, since the aggregates consist of a large number of entangled rods. 
Table 2. Magnetic energies of assemblies and critical length for different paramagnetic ions at $\mathrm{B}=2 \mathrm{~T}$ and $\mathrm{T}=298{\mathrm{~K} . \mathrm{Gd}^{3+}}^{3}$ is used in the current work.

\begin{tabular}{|c|c|c|c|}
\hline Ion & $\begin{array}{c}m_{\text {eff }}{ }^{[23]} \\
\left(\mu_{B}\right)\end{array}$ & $\begin{array}{c}U_{m, 1} \\
\left(\mathrm{~J} . m o l^{-1} \mathrm{monomer}^{-1}\right)\end{array}$ & $\begin{array}{c}L_{c} \\
(\mathrm{~nm})\end{array}$ \\
\hline $\mathrm{Nd}^{3+}$ & 3.4 & -0.29 & 2980 \\
\hline $\mathrm{Gd}^{3+}$ & 8.9 & -1.99 & 435 \\
\hline $\mathrm{Tb}^{3+}$ & 9.8 & -2.42 & 359 \\
\hline $\mathrm{Dy}^{3+}$ & 10.6 & -2.83 & 307 \\
\hline $\mathrm{Ho}^{3+}$ & 10.4 & -2.73 & 318 \\
\hline
\end{tabular}

Let us now consider how the magnetic energy affects the network structure. Under normal conditions the distribution of P1 and $\mathbf{P} 2$ species is governed by an equilibrium constant, $K_{2}$ as seen in Fig. 1a:

$$
K_{2}=e^{-\frac{\Delta G_{0}+\Delta G_{m}}{R T}}
$$

where $\Delta G_{0}$ is the Gibbs free energy at $298 \mathrm{~K}$ and $0 \mathrm{~T}$. The additional term, $\Delta G_{m}$, is a magnetic Gibbs free energy induced by the magnetic field, and is defined as $\Delta G_{m}=U_{m, \text { rod }} / 2^{[23]}$ for each rod P1 (i.e., $U_{m, r o d}=U_{m, 1} \times 700$ monomers for Gd(III)-DOTA-BTA) added to or removed from the rod network P2. This is of order $0.7 \mathrm{~kJ} \cdot \mathrm{mol}^{-1}$ at $2 \mathrm{~T}$, but does not influence the equilibrium constant as this energy is the same, to first order, for any spatial distribution of the $\mathrm{Gd}^{3+}$ ions. However, there is a small correction due to shape anisotropy:[23]

$$
K_{s}=\frac{1}{4} \mu_{0} M_{S}^{2}(1-3 N)
$$

where $N$ is the shape-dependent demagnetizing factor ( $N=0$ for an axially magnetized long rod), $M_{s}$ is the saturation magnetization that depends on distribution of the magnetized material and $\mu_{0}$ is the vacuum permeability. For the rods, $M_{s}=$ $600 \mathrm{Am}^{-1}$. The maximum anisotropy energy is therefore $0.11 \mathrm{Jm}^{-3}$. A sample volume of at least $k_{B} T / K_{s}=36 \cdot 10^{-21} \mathrm{~m}^{3}$ is therefore needed to overcome thermal energy. The necessary volume includes some thousands of rods since a single rod is $\sim 4$. $10^{-24} \mathrm{~m}^{3}$. This explains our experimental findings that magnetic field effects are only observed at the network level.

In conclusion, we have shown pronounced changes in the network topology of supramolecular polymer rods containing paramagnetic ions upon applying magnetic fields of 1-2 $\mathrm{T}$, accessible without resorting to cryogenic superconductor magnets. The magnetic energies involved are insignificant at the single rod level; they become significant only at the network scale.

In general, supramolecular polymers provide a versatile platform to assemble many magnetic ions into larger substructures and make them susceptible to the fields from modern permanent magnets or Halbach arrays. Our approach therefore opens opportunities to control and guide structure formation of supramolecular and coordination polymers using rare earth or paramagnetic ions, in order to tune collective effects in magnetic field-responsive dynamic materials.

\section{Acknowledgements}

$\mathrm{VM}, \mathrm{AS}, \mathrm{JLI}$, and TMH would like to acknowledge the funding from ANR-10-LABX-0026 CSC. GJMF received funding from Ministère de l'Education Nationale de l'Enseignement supérieur et de la Recherche. EWM acknowledges funding of the Dutch Ministry of Education, Culture and Science (Gravitation program 024.001.035). PD and TMH acknowledge the support of the Labex NIE 11-LABX-0058_NIE within the Investissement d'Avenir program ANR-10-IDEX-0002-02, the support of the University of Strasbourg Institute for Advanced Studies (USIAS) Fellowship. PD acknowledges support from SALTYSPIN ANR-17-CE09-0005
Keywords: supramolecular polymer $\bullet$ rare earth ion $\bullet$ magnet $•$ magnetic equilibrium

[1] T. Aida, E. W. Meijer, Isr. J. Chem. 2020, 60, 33-47.

[2] T. Aida, E. W. Meijer, S. I. Stupp, Science 2012, 335, 813-817.

[3] A. R. Hirst, S. Roy, M. Arora, A. K. Das, N. Hodson, P. Murray, S. Marshall, N. Javid, J. Sefcik, J. Boekhoven, J. H. Van Esch, S. Santabarbara, N. T. Hunt, R. V. Ulijn, Nature Chemistry 2010, 2, 1089-1094.

[4] M. J. Webber, C. J. Newcomb, S. I. Stupp, Soft Matter 2011, 7 , 9665-9672.

[5] A. Sorrenti, J. Leira-Iglesias, A. Sato, T. M. Hermans, Nature Communications 2017, 8, 15899.

[6] J. M. A. Carnall, C. A. Waudby, A. M. Belenguer, M. C. A. Stuart, J. J. P. Peyralans, S. Otto, Science 2010, 327, 1502-1507.

[7] J. T. Van Herpt, M. C. A. Stuart, W. R. Browne, B. L. Feringa, Langmuir 2013, 29, 8763-8767.

[8] B. Adhikari, Y. Yamada, M. Yamauchi, K. Wakita, X. Lin, K. Aratsu, T. Ohba, T. Karatsu, M. J. Hollamby, N. Shimizu, H. Takagi, R. Haruki, S. Adachi, S. Yagai, Nature Communications 2017, 8, ncomms 15254.

[9] R. Iwaura, T. Shimizu, Angewandte Chemie - International Edition 2006, 45, 4601-4604.

[10] L. Li, H. Jiang, B. W. Messmore, S. R. Bull, S. I. Stupp, Angew. Chem. Int. Ed. 2007, 46, 5873-5876.

[11] J. Zhang, R. Hao, L. Huang, J. Yao, X. Chen, Z. Shao, Chemical Communications 2011, 47, 10296-10298.

[12] H. Frisch, J. P. Unsleber, D. Lüdeker, M. Peterlechner, G. Brunklaus, M. Waller, P. Besenius, Angew. Chem. Int. Ed. 2013, 52, 10097-10101.

[13] T. J. Moyer, J. A. Finbloom, F. Chen, D. J. Toft, V. L. Cryns, S. I. Stupp, J. Am. Chem. Soc. 2014, 136, 14746-14752.

[14] E. Ohta, H. Sato, S. Ando, A. Kosaka, T. Fukushima, D. Hashizume, M. Yamasaki, K. Hasegawa, A. Muraoka, H. Ushiyama, K. Yamashita, T. Aida, Nature Chemistry 2010, 3 , 68-73.

[15] J. Leira-Iglesias, A. Sorrenti, A. Sato, P. A. Dunne, T. M. Hermans, Chemical Communications 2016, 52, 9009-9012.

[16] P. Van Der Schoot, M. E. Cates, The Journal of Chemical Physics 1994, 101, 5040-5046.

[17] T. J. Drye, M. E. Cates, The Journal of Chemical Physics 1993, 98, 9790-9797.

[18] R. Bruinsma, W. M. Gelbart, A. Ben-Shaul, The Journal of Chemical Physics 1992, 96, 7710-7727.

[19] M. S. Turner, M. E. Cates, Journal Phys: Condensed Matter 1992, 4, 3719-3741.

[20] G. Singh, H. Chan, A. Baskin, E. Gelman, N. Repnin, P. Král, R. Klajn, Science 2014, 345, 1149-1153.

[21] S. Polarz, C. Bährle, S. Landsmann, A. Klaiber, Angewandte Chemie - International Edition 2013, 52, 13665-13670.

[22] B. Yue, X. Jin, P. Zhao, M. Zhu, L. Zhu, 2019, 1804572, 1-7.

[23] J. M. D. Coey, Magnetism and Magnetic Materials, Cambridge University Press, Cambridge, UK, 2009.

[24] P. Besenius, G. Portale, P. H. H. Bomans, H. M. Janssen, A. R. A. Palmans, E. W. Meijer, PNAS 2010, 107, 1788817893.

[25] I. De Feijter, P. Besenius, L. Albertazzi, E. W. Meijer, A. R. A. Palmans, I. K. Voets, Soft Matter 2013, 9, 10025-10030.

[26] S. W. Provencher, Computer Physics Communications 1982, 27, 213-227.

[27] M. M. Tirado, C. L. Martínez, J. G. de la Torre, J. Chem. Phys. 1984, 81, 2047-2052.

[28] R. Pecora, The Journal of Chemical Physics 1968, 48, 41264128.

[29] J. A. Raper, R. Amal, Particle \& Particle Systems Characterization 1993, 10, 239-245.

[30] A. Mohraz, D. B. Moler, R. M. Ziff, M. J. Solomon, Physical Review Letters 2004, 92, 1555031-1555034.

[31] L. A. Hough, M. F. Islam, B. Hammouda, A. G. Yodh, P. A. Heiney, Nano Letters 2006, 6, 313-317.

[32] D. W. Schaefer, Science 1989, 243, 1023-1027.

[33] D. W. Schaefer, J. M. Brown, D. P. Anderson, J. Zhao, K. Chokalingam, D. Tomlin, J. Ilavsky, in Journal of Applied Crystallography, 2003, pp. 553-557. 
[34] B. J. Bauer, E. K. Hobbie, M. L. Becker, Macromolecules 2006, 39, 2637-2642.

[35] J. W. Steed, J. L. Atwood, Supramolecular Chemistry, John Wiley \& Sons, Ltd, Chichester, UK, 2009. 